\title{
EXISTENCE AND UNIQUENESS FOR THE NONSTATIONARY PROBLEM OF THE ELECTRICAL HEATING OF A CONDUCTOR DUE TO THE JOULE-THOMSON EFFECT
}

\section{XIANGSHENG XU}

\author{
Department of Mathematical Sciences \\ 301 Science-Engineering Building \\ University of Arkansas \\ Fayetteville, Arkansas 72701
}

(Received August 15, 1991 and in revised form April 10, 1992)

ABSTRACT. Existence of a weak solution is established for the initial-boundary value problem for the system $\frac{\partial}{\partial t} u-\operatorname{div}(\Theta(u) \nabla u)+\sigma(u) \alpha(u) \nabla u \nabla v=\sigma(u)|\nabla v|^{2}, \operatorname{div}(\sigma(u) \nabla v)=0$. The question of uniqueness is also considered in some special cases.

KEY WORDS AND PHRASES. Joule-Thomson effect, quadratic gradient growth in the nonlinearity, existence, uniqueness.

1991 AMS SUBJECT CLASSIFICATION CODES. 35D05, 35D10, 35K55.

\section{INTRODUCTION.}

Let $\Omega$ be a bounded domain in $R^{N}$ with smooth boundary $\partial \Omega$ and $T$ a positive number. In this paper we shall be concerned with the following problem:

$$
\begin{gathered}
u_{t}-\operatorname{div}(\Theta(u) \nabla u)+\sigma(u) \alpha(u) \nabla u \nabla v=\sigma(u)|\nabla v|^{2} \text { in } Q_{T}=\Omega \times(0, T), \\
d i v(\sigma(u) \nabla v)=0 \text { in } Q_{T}, \\
u=0 \text { on } S_{T}=\partial \Omega \times(0, T), \\
v=B(x, t) \text { on } S_{T}, \\
u(x, 0)=U_{0}(x) \text { in } \Omega \times\{0\} .
\end{gathered}
$$

Here, $\Theta(u), \sigma(u)$, and $\alpha(u)$ are known functions of their argument and $B, U_{0}$ are given data.

Problem (1.1) may be proposed as a model for the electrical heating of a conductor resulted from Thomson's effect and Joule's heating; see [1]. In this situation, $u$ is the temperature of the conductor and $v$ the effective potential. Equation (1.1b) represents the conservation of charge, while (1.1a) says that there are two types of heat source involved in the heat conduction; the convective term in (1.1a) corresponds to Thomson's effect and the quadratic term in (1.1a) reflects Joule's heating.

If $N=2, \alpha=0$, and $\sigma \in C^{1}(\mathbf{R})$ is such that

$$
0<m \leq \sigma(s) \leq \boldsymbol{M}, s \in \mathbf{R}
$$

for some $M \geq m$ the existence of a weak solution is established for (1.1) in [2]. A result due to Shi, 
Shillor, and $\mathrm{Xu}$ [3] asserts that the assumption that $N=2$ and $\sigma \in C^{1}(R)$ in [2] can be eliminated. The associated stationary problem of (1.1) was first considered in [1] where $\sigma$ and $\Theta$ are assumed to obey the Wiedemann-Franz law, i.e.,

$$
\frac{\sigma(u)}{\Theta(u)}=\frac{c}{u} \text { for some } c>0,
$$

and $\alpha$ is assumed to be linear. Under these assumptions the stationary problem can be reformulated as

$$
\begin{aligned}
& \left.\begin{array}{l}
\operatorname{div}(A(u, v) \nabla u)=0 \\
\operatorname{div}(A(u, v) \nabla v)=0
\end{array}\right\} \text { in } \Omega \\
& u=u_{0}, v=v_{0} \text { on } \partial \Omega .
\end{aligned}
$$

Thus a uniform bound for the temperature can be obtained, thereby establishing an existence assertion. See [1] for details.

Our main objective is to prove an existence theorem for (1.1) under rather general assumptions on the data. Indeed, if the temperature is known to be bounded, our assumptions are much weaker than those in [1]. Of course, our approach is also different and is based upon an approximation scheme. We also consider the question of uniqueness, but we are only able to show that the uniqueness holds when $N=2$ and $\Theta(s)=s$.

The mathematical interest of our problem is due to the presence of quadratic gradient growth in the nonlinearity. In general, nonlinearities of this nature render the classical regularity and compactness results useless; see [4] for a detailed description in this regard. Our method makes full use of the explicit nonlinear structure of our problem, which enables us to extract enough extra information to obtain an existence assertion. We refer the reader to [4] for more related works in this direction.

Finally, let us make some comments on notation. The letter $c$ will be used to denote the generic constant. When distinction among different constants is needed, we add a subscript $i \in\{0,1,2, \ldots\}$ to $c$. Other notation conventions follow those employed in [5] and [6]. For example,

$$
\|f\|_{p, \Omega}=\|f\|_{p}=\left(\int_{\Omega}|f|^{p} d x\right)^{1 / p}
$$

for $f \in L^{p}(\Omega)$.

\section{EXISTENCE}

In this section we first establish an existence assertion for the associated stationary problem. Then a weak solution to (1.1) is obtained via the implicit discretization in time.

Let $\Omega$ be a bounded domain in $\mathbf{R}^{N}$ with smooth boundary $\partial \Omega$. Consider the system

$$
\left.\begin{array}{l}
\begin{array}{l}
-\operatorname{div}(I(u, v) \nabla u)+K(u+v)=J(u+v)|\nabla v|^{2}+H(x) \\
-\operatorname{div}(J(u+v) \nabla v)=0
\end{array}
\end{array}\right\} \text { in } \Omega
$$

coupled with boundary conditions

$$
u=u_{0} \text { on } \partial \Omega, v=v_{0} \text { on } \partial \Omega .
$$

With respect to the data involved, we assume the following. 
(H1) $I(s, \tau), J(s), K(s)$ are all continuous;

(H2) There exist two positive numbers $m \leq M$ such that

$$
m \leq I(s, \tau) \leq M, m \leq J(s) \leq M
$$

for all $s, \tau \in \mathbf{R}$

(H3) $K$ is nondecreasing and satisfies

$$
|K(s)| \leq c|s| \text { for some } c>0 ;
$$

(H4) $u_{0} \in W^{1,2}(\Omega), v_{0} \in W^{1,2}(\Omega) \cap L^{\infty}(\Omega)$, and $H \in L^{2}(\Omega)$.

A weak solution to $(2.1)$ is defined as a pair $(u, v)$ such that

$$
\begin{gathered}
u, v \in W^{1,2}(\Omega), \\
\int_{\Omega} I(u, v) \nabla u \nabla \xi d x+\int_{\Omega} K(u+v) \xi d x=\int_{\Omega}\left(J(u+v)|\nabla v|^{2}+H(x)\right) \xi d x
\end{gathered}
$$

for all $\xi \in W_{0}^{1,2}(\Omega) \cap L^{\infty}(\Omega)$,

$$
\begin{gathered}
\int_{\Omega} J(u+v) \nabla v \nabla \eta d x=0 \text { for all } \eta \in W_{0}^{1,2}(\Omega), \\
u=u_{0}, v=v_{0} \text { on } \partial \Omega .
\end{gathered}
$$

THEOREM 2.1. Let (H1) - (H4) be satisfied. Then there exists a weak solution to (2.1). PROOF. For each $k$ define

$$
\begin{gathered}
P_{k}(x)= \begin{cases}k & \text { if }|x|^{2} \geq k, \\
|x|^{2} & \text { if }|x|^{2}<k,\end{cases} \\
K_{k}(s)=\left\{\begin{aligned}
k & \text { if } K(s)>k, \\
K(s) & \text { if }|K(s)| \leq k \\
-k & \text { if } K(s)<-k .
\end{aligned}\right.
\end{gathered}
$$

Denote by $V$ the product space $W^{1,2}(\Omega) \times W^{1,2}(\Omega)$ and $V^{*}$ its topological dual. Set $E=\left\{\left(u_{1}, v_{1}\right) \in V:\left.u_{1}\right|_{\partial \Omega}=u_{0}\right.$ and $\left.\left.v_{1}\right|_{\partial \Omega}=v_{0}\right\}$. Clearly, $E$ is a closed, convex subset of $V$. For each $k$ define an operator $A_{k}: E \rightarrow V^{*}$ by

$$
\begin{aligned}
\left(A_{k}\left(w_{1}\right), w_{2}\right)= & \int_{\Omega} I\left(u_{1}, v_{1}\right) \nabla u_{1} \nabla u_{2} d x+\int_{\Omega}\left\{K_{k}\left(u_{1}+v_{1}\right)-J\left(u_{1}+v_{1}\right) P_{k}\left(\nabla v_{1}\right)\right. \\
& -H(x)\} u_{2} d x \\
& +\int_{\Omega} J\left(u_{1}+v_{1}\right) \nabla v_{1} \nabla v_{2} d x \\
w_{1}= & \left(u_{1}, v_{1}\right) \in E, w_{2}=\left(u_{2}, v_{2}\right) \in V
\end{aligned}
$$


where $(\cdot, \cdot)$ denotes the duality pairing between $V^{*}$ and $V$. By the definition of $P_{k}, A_{k}$ is welldefined. It is not difficult to verify that for each $k, A_{k}$ satisfies the following conditions:

(i) $A_{k}$ is bounded.

(ii) $A_{k}$ is pseudomonotone.

(iii) $\left(A_{k}(w), w-w_{0}\right) /\|w\|_{v} \rightarrow \infty$ as $\|w\|_{v} \rightarrow \infty$ for $w \in E$, where $w_{0}=\left(u_{0}, v_{0}\right)$.

Now we are in a position to invoke an existence result in [7, p. 169] to conclude that for each $k$ there exists at least one vector-valued function $w_{k}=\left(u_{k}, v_{k}\right) \in E$ such that

$$
\left(A_{k}\left(w_{k}\right), w-w_{k}\right) \geq 0 \text { for all } w \in E .
$$

This is easily seen to be equivalent to the following statements:

$$
\begin{gathered}
\left.u_{k}\right|_{\partial \Omega}=u_{0},\left.v_{k}\right|_{\partial \Omega}=v_{0} \\
\int_{\Omega} I\left(u_{k}, v_{k}\right) \nabla u_{k} \nabla \xi d x+\int_{\Omega} K_{k}\left(u_{k}+v_{k}\right) \xi d x=\int_{\Omega}\left(J\left(u_{k}+v_{k}\right) P_{k}\left(\nabla v_{k}\right)+H(x)\right) \xi d x \\
\int_{\Omega} J\left(u_{k}+v_{k}\right) \nabla v_{k} \nabla \xi d x=0,
\end{gathered}
$$

for all $\xi \in W_{0}^{1,2}(\Omega)$. Equation (2.8) allows us to use the weak maximum principle to get

$$
\sup _{x \in \Omega}\left|v_{k}(x)\right| \leq c(k=1,2, \ldots) .
$$

Set $\xi=v_{k}-v_{0}$ in $(2.8)$ to deduce

$$
\left\|\nabla v_{k}\right\|_{2} \leq c(k=1,2, \ldots)
$$

Let $\xi=u_{k}-u_{0}$ in (2.7) to derive

$$
\begin{gathered}
\int_{\Omega} I\left(u_{k}, v_{k}\right)\left|\nabla u_{k}\right|^{2} d x+\int_{\Omega} K_{k}\left(u_{k}+v_{k}\right)\left(u_{k}-u_{0}\right) d x \\
=\int_{\Omega} I\left(u_{k}, v_{k}\right) \nabla u_{k} \nabla u_{0} d x+\int_{\Omega} J\left(u_{k}+v_{k}\right) P_{k}\left(\nabla v_{k}\right)\left(u_{k}-u_{0}\right) d x+\int_{\Omega} H(x)\left(u_{k}-u_{0}\right) d x .
\end{gathered}
$$

We estimate, with the aid of (H3) and (2.9), that

$$
\begin{aligned}
\int_{\Omega} K_{k}\left(u_{k}+v_{k}\right)\left(u_{k}-u_{0}\right) d x & \\
& =\int_{\Omega} K_{k}\left(u_{k}+v_{k}\right)\left(u_{k}+v_{k}\right) d x-\int_{\Omega} K_{k}\left(u_{k}+v_{k}\right)\left(v_{k}+u_{0}\right) d x \\
& \geq-\left\|K_{k}\left(u_{k}+v_{k}\right)\right\|_{2}\left\|v_{k}+u_{0}\right\|_{2} \\
& \geq-c_{1}\left\|u_{k}\right\|_{2}-c_{2} .
\end{aligned}
$$

For each positive integer $j$ define

$$
L_{j}(s)=\left\{\begin{array}{c}
j \text { if } s \geq j \\
s \text { if }|s|<j \\
-j \text { if } s \geq-j
\end{array}\right.
$$


We calculate, using (2.8), that

$$
\begin{aligned}
& \int_{\Omega} J\left(u_{k}+v_{k}\right) P_{k}\left(\nabla v_{k}\right) L_{j}\left(u_{k}-u_{0}\right) d x \\
& \quad=\int_{\Omega} J\left(u_{k}+v_{k}\right) P_{k}\left(\nabla v_{k}\right)\left(\left[L_{j}\left(u_{k}-u_{0}\right)\right]^{+}-\left[L_{j}\left(u_{k}-u_{0}\right)\right]^{-}\right) d x \\
& \leq \int_{\Omega} J\left(u_{k}+v_{k}\right) P_{k}\left(\nabla v_{k}\right)\left[L_{j}\left(u_{k}-u_{0}\right)\right]^{+} d x \\
& \leq \int_{\Omega} J\left(u_{k}+v_{k}\right)\left|\nabla v_{k}\right|^{2}\left[L_{j}\left(u_{k}-u_{0}\right)\right]^{+} d x \\
&=\int_{\Omega} J\left(u_{k}+v_{k}\right) \nabla v_{k}\left\{\nabla\left(v_{k}\left[L_{j}\left(u_{k}-u_{0}\right)\right]^{+}\right)-v_{k} \nabla\left[L_{j}\left(u_{k}-u_{0}\right)\right]+\right\} d x \\
&=\int_{\Omega} J\left(u_{k}+v_{k}\right) \nabla v_{k} v_{k} \nabla\left[L_{j}\left(u_{k}-u_{0}\right)\right]^{+} d x \\
& \leq c\left\|\nabla v_{k}\right\|_{2}\left\|\nabla\left(u_{k}-u_{0}\right)\right\|_{2} \leq c_{1}\left\|\nabla u_{k}\right\|_{2}+c_{2} .
\end{aligned}
$$

Send $j$ to infinity to get

$$
\int_{\Omega} J\left(u_{k}+v_{k}\right) P_{k}\left(\nabla v_{k}\right)\left(u_{k}-u_{0}\right) d x \leq c_{1}\left\|\nabla u_{k}\right\|_{2}+c_{2}
$$

Use this and (2.12) in (2.11) to obtain

$$
m \int_{\Omega}\left|\nabla v_{k}\right|^{2} d x \leq c_{1}\left\|\nabla u_{k}\right\|_{2}+c_{2}\left\|u_{k}\right\|_{2}+c_{3}(k=1,2, \ldots) .
$$

According to Poincaré's inequality,

$$
\left\|u_{k}-u_{0}\right\|_{2} \leq c\left\|\nabla\left(u_{k}-u_{0}\right)\right\|_{2} \leq c\left(\left\|\nabla u_{k}\right\|_{2}+\left\|\nabla u_{0}\right\|_{2}\right) .
$$

Consequently,

$$
\left\|u_{k}\right\|_{2} \leq\left\|u_{k}-u_{0}\right\|_{2}+\left\|u_{0}\right\|_{2} \leq c_{1}\left\|\nabla u_{k}\right\|_{2}+c_{2} .
$$

Combining (2.13) and (2.14) yields

$$
\left\|u_{k}\right\|_{2}+\left\|\nabla u_{k}\right\|_{2} \leq c(k=1,2, \ldots) .
$$

In view of (2.9), (2.10), and (2.15), we may assume that there exists a subsequence of $\{k\}$, still denoted by $\{k\}$, such that

$$
\begin{aligned}
& v_{k} \rightarrow v \text { weakly in } W^{1,2}(\Omega) \text { and strongly in } L^{2}(\Omega), \\
& u_{k} \rightarrow u \text { weakly in } W^{1,2}(\Omega) \text { and strongly in } L^{2}(\Omega) .
\end{aligned}
$$

Then it immediately follows from (H1), (H2), and (H3) that

$$
I\left(u_{k}, v_{k}\right) \rightarrow I(u, v), K_{k}\left(u_{k}+v_{k}\right) \rightarrow K(u+v)
$$

and

$$
J\left(u_{k}+v_{k}\right) \rightarrow J(u+v) \text { strongly in } L^{2}(\Omega)
$$


Set $\xi=v_{k}-v$ in $(2.8)$ to deduce

$$
\begin{gathered}
\lim _{k \rightarrow \infty} \sup m \int_{\Omega}\left|\nabla\left(v_{k}-v\right)\right|^{2} d x \leq \limsup _{k \rightarrow \infty} \int_{\Omega} J\left(u_{k}+v_{k}\right)\left|\nabla\left(v_{k}-v\right)\right|^{2} d x \\
=\lim _{k \rightarrow \infty}-\int_{\Omega} J\left(u_{k}+v_{k}\right) \nabla v \nabla\left(v_{k}-v\right) d x=0 .
\end{gathered}
$$

This implies that

$$
P_{k}\left(\nabla v_{k}\right) \rightarrow|\nabla v|^{2} \text { strongly in } L^{1}(\Omega) .
$$

Then the theorem follows from taking $k \rightarrow \infty$ in (2.7) and (2.8).

Let $\Omega, H(x), u_{0}, v_{0}$ be given as before. Consider the following problem:

$$
\begin{gathered}
\left.\begin{array}{c}
u-\operatorname{div}(\Theta(u) \nabla u)+\sigma(u) \beta^{\prime}(u) \nabla u \nabla v=\sigma(u)|\nabla v|^{2}+H(x) \\
\operatorname{div}(\sigma(u) \nabla v)=0
\end{array}\right\} \text { in } \Omega, \\
\left.u\right|_{\partial \Omega}=u_{0},\left.v\right|_{\partial \Omega}=v_{0} .
\end{gathered}
$$

We impose the following conditions on $\Theta, \sigma, \beta$ :

(H5) $\Theta, \sigma, \beta$ are continuous and satisfy

$$
m \leq \Theta(s) \leq M, m \leq \sigma(s) \leq M, m \leq \beta(s) \leq M \text { for some } M \geq m>0
$$

for all $s \in \mathbf{R}$.

(H6) $\beta^{\prime}$ is continuous and bounded.

A weak solution to (2.21) can be defined in the same manner as that to (2.1).

THEOREM 2.2. Under the above assumptions there is a weak solution to (2.21).

PROOF. Let

$$
F(s)=\int_{0}^{s} \frac{\Theta(\tau)}{\sigma(\tau) \beta(\tau)} d \tau .
$$

Then by (H5) there exists two positive constants $c_{1}, c_{2}$ such that

$$
0<c_{1} \leq F^{\prime}(s) \leq c_{2} \text { for all } s \in R .
$$

Denote by $K$ the inverse of $F$. From (2.22), we have

$$
c_{4} \leq K^{\prime}(s) \leq c_{3} \text { for all } s \in R
$$

for some $c_{3} \geq c_{4}>0$. Thus $K$ satisfies (H3). Now set

$$
\begin{gathered}
I(s, \tau)=\sigma(K(s+\tau) \beta(K(s+\tau)), \\
J(s)=\sigma(K(s)) .
\end{gathered}
$$

Clearly, $I, J, K$ satisfy (H1)-(H3). By Theorem 2.1, there is a weak solution to the following problem:

$$
\left.\begin{array}{c}
-\operatorname{div}(I(a, b) \nabla a)+K(a+b)=J(a+b)|\nabla b|^{2}+H(x) \\
-\operatorname{div}(J(a+b) \nabla b)=0
\end{array}\right\} \text { in } \Omega
$$




$$
\left.a\right|_{\partial \Omega}=F\left(u_{0}\right)-v_{0},\left.b\right|_{\partial \Omega}=v_{0} .
$$

Let $u=K(a+b), v=b$. We wish to show that $(u, v)$ thus defined is a weak solution to (2.21). Clearly, $u, v \in W^{1,2}(\Omega)$, and $(2.21 \mathrm{~b})$ is satisfied. Note that

$$
a=F(u)-b=F(u)-v .
$$

We derive from $(2.23),(2.24)$, and $(2.25)$ that

$$
\begin{aligned}
-\operatorname{div}(\sigma(u) \beta(u) & \nabla(F(u)-v))+u \\
= & -\operatorname{div}\left(\sigma(u) \beta(u) F^{\prime}(u) \nabla u-\sigma(u) \beta(u) \nabla v\right)+u \\
= & -\operatorname{div}(\Theta(u) \nabla u)+\operatorname{div}(\sigma(u) \beta(u) \nabla v)+u=\sigma(u)|\nabla v|^{2}+H(x) \text { in } \Omega .
\end{aligned}
$$

We conclude from (2.24) and (2.26) that

$$
-\operatorname{div}(\sigma(u) \nabla v)=0 \text { in } \Omega .
$$

We calculate from $(2.28)$ that for any $\xi \in W_{0}^{1,2}(\Omega) \cap L^{\infty}(\Omega)$,

$$
\begin{aligned}
\int_{\Omega} \sigma(u) \beta(u) \nabla v \nabla \xi d x & =\int_{\Omega} \sigma(u) \nabla v(\nabla(\beta(u) \xi)-\xi \nabla \beta(u)) d x \\
& =-\int_{\Omega} \sigma(u) \beta^{\prime}(u) \nabla u \nabla v \xi d x .
\end{aligned}
$$

Thus

$$
\operatorname{div}(\sigma(u) \beta(u) \nabla v)=\sigma(u) \beta^{\prime}(u) \nabla u \nabla v
$$

in the sense of distributions. Use this in (2.7) to obtain the theorem.

REMARK. In fact, we only need to assume that $\beta$ is bounded. Then we can always select a number $c$ large enough so that

$$
0<m \leq c+\beta \leq M .
$$

Also, if we know that $u$ is bounded a priori, then there is no need to assume that $\Theta, \sigma, \beta$ are bounded above. In this sense, our hypotheses are much weaker than those in [1]. However, in the generality considered here it does not seem likely that $u$ can be bounded.

Now we are ready to prove an existence assertion for the following problem:

$$
\begin{aligned}
& \left.\begin{array}{l}
\frac{\partial u}{\partial t}-\operatorname{div}(\Theta(u) \nabla u)+\sigma(u) \beta^{\prime}(u) \nabla u \nabla v=\sigma(u)|\nabla v|^{2} \\
\operatorname{div}(\sigma(u) \nabla v)=0
\end{array}\right\} \in Q_{T} \equiv \Omega x(0, T) \\
& u=0, v=B \text { on } S_{T}=\partial \Omega \times(0, T), \\
& u=U_{0} \text { on } \Omega \times\{0\} \text {. }
\end{aligned}
$$

THEOREM 2.3. Let $\Omega, \Theta, \sigma, \beta$ be given as before. Assume that $B \in L^{2}\left(0, T ; W^{1,2}(\Omega)\right) \cap L^{\infty}\left(Q_{T}\right)$, and $U_{0} \in W^{1,2}(\Omega)$. Then there exists a weak solution to $(2.30)$, i.e., there is a pair $(u, v)$ such that

$$
\begin{gathered}
u, v \in L^{2}\left(0, T ; W^{1,2}(\Omega)\right), \\
u, v-B \in L^{2}\left(0, T ; W_{0}^{1,2}(\Omega)\right),
\end{gathered}
$$




$$
\begin{gathered}
-\int_{Q_{T}} u \xi_{t} d x d t+\int_{Q_{T}} \Theta(u) \nabla u \nabla \xi d x d t+\int_{Q_{T}} \sigma(u) \beta^{\prime}(u) \nabla u \nabla v \xi d x d t \\
=\int_{Q_{T}} \sigma(u)|\nabla v|^{2} \xi d x d t+\int_{\Omega} U_{0}(x) \xi(x, 0) d x
\end{gathered}
$$

for all $\xi \in H^{1}\left(0, T ; W_{0}^{1,2}(\Omega)\right) \cap L^{\infty}\left(Q_{T}\right)$ such that $\xi(x, T) \equiv 0$,

$$
\int_{Q_{T}} \sigma(u) \nabla v \nabla \eta d x d t=0 \text { for all } \eta \in L^{2}\left(0, T ; W_{0}^{1,2}(\Omega)\right) .
$$

PROOF. We shall follow the approach presented in [3] using a discretization technique. Let $n \in\{1,2, \ldots\}$. Set $\delta=T / n$. For $k=1,2, \ldots, n$, denote by $B_{n}^{(k)}$ the integral $\frac{1}{\delta} \int_{(k-1) \delta}^{k \delta} B(x, \tau) d \tau$. Subsequently, we may generate a set of $n$ pairs $\left(u_{n}^{(1)}, v_{n}^{(1)}\right), \ldots,\left(u_{n}^{(n)}, v_{n}^{(n)}\right)$ via the following iteration formula:

$$
\begin{gathered}
\frac{u_{n}^{(k)}-u_{n}^{(k-1)}}{\delta}-\operatorname{div}\left(\Theta\left(u_{n}^{(k)} \nabla u_{n}^{(k)}\right)+\sigma\left(u_{n}^{(k)}\right) \beta^{\prime}\left(u_{n}^{(k)}\right) \nabla u_{n}^{(k)} \nabla v_{n}^{(k)}\right. \\
=\sigma\left(u_{n}^{(k)}\right)\left|\nabla v_{n}^{(k)}\right|^{2} \text { in } \Omega, \\
\operatorname{div}\left(\sigma\left(u_{n}^{(k)}\right) \nabla v_{n}^{(k)}\right)=0 \text { in } \Omega, \\
u_{n}^{(k)}=0 \text { on } \partial \Omega, \\
v_{n}^{(k)}=B_{n}^{(k)} \text { on } \partial \Omega, \\
k=1,2, \ldots n,
\end{gathered}
$$

where

$$
u_{n}^{(0)}=U_{0}
$$

Define two function $u_{n}, v_{n}$ by

$$
\begin{aligned}
& u_{n}(x, t)= \begin{cases}U_{0} & \text { if } t \leq 0, \\
u_{n}^{(k)} & \text { if }(k-1) \delta<t \leq k \delta(k=1, \ldots, n),\end{cases} \\
& v_{n}(x, t)=\left\{\begin{array}{l}
v_{n}^{(1)} \text { if } t \leq \delta, \\
v_{n}^{(k)} \text { if }(k-1) \delta<t \leq k \delta(k=2, \ldots, n) .
\end{array}\right.
\end{aligned}
$$

We infer from (2.36) that

$$
\begin{gathered}
\sup _{(x, t) \in Q_{T}}\left|v_{n}(x, t)\right| \leq c, \\
\left\|\nabla v_{n}\right\|_{2, Q_{T}} \leq c(n=1,2, \ldots) .
\end{gathered}
$$

Let $L_{j}(s)$ be given as before. Note that

$$
\int_{\Omega} \sigma\left(u_{n}^{(k)}\right) \beta^{\prime}\left(u_{n}^{(k)}\right) \nabla u_{n}^{(k)} \nabla v_{n}^{(k)} L_{j}\left(u_{n}^{(k)}\right) d x
$$




$$
\begin{aligned}
& =-\int_{\Omega} \sigma\left(u_{n}^{(k)}\right) \nabla v_{n}^{(k)} \beta\left(u_{n}^{(k)}\right) \nabla L_{j}\left(u_{n}^{(k)}\right) d x \\
& \leq c\left\|\nabla v_{n}^{(k)}\right\|_{2}\left\|\nabla u_{n}^{(k)}\right\|_{2},
\end{aligned}
$$

and that

$$
\begin{gathered}
\int_{\Omega} \sigma\left(u_{n}^{(k)}\right)\left|\nabla v_{n}^{(k)}\right|^{2} L_{j}\left(u_{n}^{(k)}\right) d x \\
=-\int_{\Omega} \sigma\left(u_{n}^{(k)}\right) v_{n}^{(k)} \nabla v_{n}^{(k)} \nabla L_{j}\left(u_{n}^{(k)}\right) d x \\
\leq c\left\|\nabla v_{n}^{(k)}\right\|_{2}\left\|\nabla u_{n}^{(k)}\right\|_{2} .
\end{gathered}
$$

Multiply (2.35) by $L_{j}\left(u_{n}^{(k)}\right)$ and use (2.39) and (2.40) in the resulting equation to obtain

$$
\begin{gathered}
\frac{1}{\delta} \int_{\Omega}\left(u_{n}^{(k)}-u_{n}^{(k-1)}\right) L_{j}\left(u_{n}^{(k)}\right) d x+\int_{\Omega} \Theta\left(u_{n}^{(k)}\right) \nabla u_{n}^{(k)} \nabla L_{j}\left(u_{n}^{(k)}\right) d x \\
\leq c\left\|\nabla v_{n}^{(k)}\right\|_{2}\left\|\nabla u_{n}^{(k)}\right\|_{2} .
\end{gathered}
$$

Observe that

$$
\left(u_{n}^{(k)}-u_{n}^{(k-1)}\right) u_{n}^{(k)} \geq \frac{1}{2}\left(u_{n}^{(k)}\right)^{2}-\frac{1}{2}\left(u_{n}^{(k-1)}\right)^{2} .
$$

Send $j$ to infinity in (2.41) and use (2.42) in the resulting equation to obtain

$$
\frac{1}{\delta} \int_{\Omega}\left\{\frac{1}{2}\left(u_{n}^{(k)}\right)^{2}-\frac{1}{2}\left(u_{n}^{(k-1)}\right)^{2}\right\} d x+\frac{m}{2} \int_{\Omega}\left|\nabla u_{n}^{(k)}\right|^{2} d x \leq c\left\|\nabla v_{n}^{(k)}\right\|_{2}^{2}
$$

Pick up an $\ell$ from $\{1, \ldots, n\}$ and sum for $k=1, \ldots, \ell$ to deduce

$$
\begin{gathered}
\frac{1}{2} \int_{\Omega} u_{n}^{2}(x, \ell \delta) d x+\frac{m}{2} \int_{0}^{\ell \delta} \int_{\Omega}\left|\nabla u_{n}\right|^{2} d x d t \\
\leq c \int_{0}^{\ell \delta} \int_{\Omega}\left|\nabla v_{n}\right|^{2} d x d t \\
+\frac{1}{2} \int_{\Omega} U_{0}^{2}(x) d x \leq c_{1} .
\end{gathered}
$$

Consequently,

$$
\sup _{0 \leq t \leq T} \int_{\Omega} u_{n}^{2}(x, t) d x+\int_{0}^{T} \int_{\Omega}\left|\nabla u_{n}\right|^{2} d x d t \leq c
$$

In view of (2.39) and (2.40), we may rewrite (2.35) to read 


$$
\begin{gathered}
\frac{u_{n}^{(k)}-u_{n}^{(k-1)}}{\delta}-\operatorname{div}\left(\Theta\left(u_{n}^{(k)}\right) \nabla u_{n}^{(k)}\right)+\operatorname{div}\left(\sigma\left(u_{n}^{(k)}\right) \beta\left(u_{n}^{(k)}\right) \nabla v_{n}^{(k)}\right) \\
=\operatorname{div}\left(\sigma\left(u_{n}^{(k)}\right) v_{n}^{(k)} \nabla u_{n}^{(k)}\right) \text { in } \Omega .
\end{gathered}
$$

For each $n$ define $\overline{u_{n}}(x, t)$ by

$$
\overline{u_{n}}(x, t)=\frac{t-(k-1) \delta}{\delta} u_{n}^{(k)}+\frac{k \delta-t}{\delta} u_{n}^{(k-1)} \text { if }(k-1) \delta<t \leq k \delta, k=1,2, \ldots, n .
$$

We deduce from (2.45) that

$$
\begin{gathered}
\frac{\partial}{\partial t} \overline{u_{n}}-\operatorname{div}\left(\Theta\left(u_{n}\right) \nabla u_{n}\right)+\operatorname{div}\left(\sigma\left(u_{n}\right) \beta\left(u_{n}\right) \nabla v_{n}\right) \\
=\operatorname{div}\left(\sigma\left(u_{n}\right) v_{n} \nabla v_{n}\right)
\end{gathered}
$$

in $L^{2}\left(0, T ; W^{-1,2}(\Omega)\right)$.

In view of (2.44), (2.37) and (2.38), we obtain that $\left\{\frac{\partial}{\partial t} \bar{u}_{n}\right\}$ is bounded in $L^{2}\left(0, T ; W^{-1,2},(\Omega)\right)$. This allows us to invoke Lions-Aubin's theorem to conclude that

$$
\left\{\overline{u_{n}}\right\} \text { is precompact in } L^{2}\left(Q_{T}\right) \text {. }
$$

Use $u_{n}^{(k)}-u_{n}^{(k-1)}$ as a test function in (2.45) to get

$$
\begin{aligned}
& \frac{1}{\delta} \int_{\Omega}\left(u_{n}^{(k)}-u_{n}^{(k-1)}\right)^{2} d x \\
& =\int_{\Omega}\left(-\Theta\left(u_{n}^{(k)}\right) \nabla u_{n}^{(k)}+\sigma\left(u_{n}^{(k)}\right) \beta\left(u_{n}^{(k)}\right) \nabla v_{n}^{(k)}\right. \\
& \left.\quad-\sigma\left(u_{n}^{(k)}\right) v_{n}^{(k)} \nabla v_{n}^{(k)}\right) \nabla\left[u_{n}^{(k)}-u_{n}^{(k-1)}\right] d x \\
& \leq c\left(\left\|\nabla u_{n}^{(k)}\right\|_{2}+\left\|\nabla v_{n}^{(k)}\right\|_{2}\right)\left(\left\|\nabla u_{n}^{(k)}\right\|_{2}+\left\|\nabla u_{n}^{(k-1)}\right\|_{2}\right) .
\end{aligned}
$$

Note that

$$
\left\|\nabla u_{n}^{(k)}\right\|_{2}=\frac{1}{\delta^{1 / 2}}\left(\int_{(k-1) \delta}^{k \delta} \int_{\Omega}\left|\nabla u_{n}\right|^{2} d x d t\right)^{1 / 2} \leq \frac{c}{\delta^{1 / 2}}
$$

Similarly,

$$
\left\|\nabla v_{n}^{(k)}\right\|_{2} \leq \frac{c}{\delta^{1 / 2}}
$$

Use (2.49) and (2.50) in (2.48) and then sum for $k=1, \ldots, n$ to derive

$$
\int_{0}^{T} \int_{\Omega}\left(u_{n}(x, t)-u_{n}(x, t-\delta)\right)^{2} d x \leq c \delta^{1 / 2}
$$

On the other hand,

$$
\int_{Q_{T}}\left(u_{n}-\overline{u_{n}}\right)^{2} d x d t=\frac{1}{3} \int_{0}^{T} \int_{\Omega}\left(u_{n}(x, t)-u_{n}(x, t-\delta)\right)^{2} d x d t
$$




$$
\leq c \delta^{1 / 2} \rightarrow 0 \text { as } n \rightarrow \infty \text {. }
$$

Thus $\left\{u_{n}\right\}$ is also precompact in $L^{2}\left(Q_{T}\right)$.

There exists a subsequence of $\{n\}$, still denoted by $\{n\}$, such that

$$
\begin{gathered}
u_{n} \rightarrow u \text { strongly in } L^{2}\left(Q_{T}\right) \text { and weakly in } L^{2}\left(0, T ; W^{1,2}(\Omega)\right) \text {, and } \\
v_{n} \rightarrow v \text { weakly in } L^{2}\left(0, T ; W^{1,2}(\Omega)\right) .
\end{gathered}
$$

To pass to the limit in (2.46), we still need to show that

$$
v_{n} \rightarrow v \text { strongly in } L^{2}\left(Q_{T}\right)
$$

We infer from (2.36) that

$$
\int_{Q_{T}} \sigma\left(u_{n}\right) \nabla v_{n} \nabla \xi d x d t=0 \text { for all } \xi \in L^{2}\left(0, T ; W_{0}^{1,2}(\Omega)\right) .
$$

Let $B_{n}(x, t)=B_{n}^{(k)}(x)$ if $(k-1) \delta<t \leq k \delta, k=1,2, \ldots, n$. It is easy to see that

$$
B_{n} \rightarrow B \text { strongly in } L^{2}\left(0, T ; W^{1,2}(\Omega)\right) \text {. }
$$

Set $\xi=v_{n}-v-B_{n}+B$ in (2.52) to deduce

$$
\begin{gathered}
\int_{Q_{T}} \sigma\left(u_{n}\right)\left|\nabla\left(v_{n}-v\right)\right|^{2} d x d t=\int_{Q_{T}} \sigma\left(u_{n}\right) \nabla v_{n} \nabla\left(B_{n}-B\right) d x d t \\
-\int_{Q_{T}} \sigma\left(u_{n}\right) \nabla v \nabla\left(v_{n}-v\right) d x d t \\
\rightarrow 0 \text { as } n \rightarrow \infty
\end{gathered}
$$

Consequently, we have

$$
v_{n} \rightarrow v \text { strongly in } L^{2}\left(0, T ; W^{1,2}(\Omega)\right)
$$

Thus (2.51) follows. Now we can take $n \rightarrow \infty$ in (2.46) to get

$$
\begin{gathered}
\frac{\partial}{\partial t} u-\operatorname{div}(\Theta(u))+\operatorname{div}(\sigma(u) \beta(u) \nabla v)=\operatorname{div}(\sigma(u) v \nabla v) \\
\in L^{2}\left(0, T ; W^{-1,2}(\Omega)\right) .
\end{gathered}
$$

Send $n$ to infinity in (2.52) to get (2.34). Then it is easy to verify from (2.34) that

$$
\begin{gathered}
\operatorname{div}(\sigma(u) \beta(u) \nabla v)=\sigma(u) \beta^{\prime}(u) \nabla u \nabla v, \\
\operatorname{div}(\sigma(u) v \nabla v)=\sigma(u)|\nabla v|^{2}
\end{gathered}
$$

in the sense of distributions. Use (2.55) and (2.56) in (2.54) to obtain (2.33). The proof is complete.

3. UNIQUENESS.

In this section, a uniqueness assertion is established for (1.1) in some special cases. 
THEOREM 3.1. Let the assumptions of Theorem 2.3 hold. Assume that $\Theta(s)=s$ and that $\sigma$ is Lipschitz continuous. Then there exists at most one solution to (1.1) in the space $L^{2}\left(0, T ; W_{0}^{1,2}(\Omega)\right) \times L^{\infty}\left(0, T ; W^{1, \infty}(\Omega)\right)$.

PROOF. Suppose that there exist two solutions $\left(u_{1}, v_{1}\right)$ and $\left(u_{2}, v_{2}\right)$ to $(1.1)$. First note that (2.54) is equivalent to (2.33) when (2.34) holds true. Set

We derive from (2.54) that

$$
\bar{u}=u_{1}-u_{2}, \bar{v}=v_{1}-v_{2} .
$$

$$
\begin{gathered}
\frac{\partial}{\partial t} \bar{u}-\Delta \bar{u}=\operatorname{div}\left(\sigma\left(u_{1}\right) v_{1} \nabla v_{1}-\sigma\left(u_{2}\right) v_{2} \nabla v_{2}\right)-\operatorname{div}\left(\sigma\left(u_{1}\right) \beta\left(u_{1}\right) \nabla v_{1}-\sigma\left(u_{2}\right) \beta\left(u_{2}\right) \nabla v_{2}\right) \\
\text { in } L^{2}\left(0, T ; W^{-1,2}(\Omega)\right) .
\end{gathered}
$$

Thus,

$$
\begin{aligned}
\frac{1}{2}\|\bar{u}(x, t)\|_{2, \Omega}^{2} & +\int_{0}^{t} \int_{\Omega}|\nabla \bar{u}|^{2} d x d \tau=\int_{0}^{t} \int_{\Omega}\left(\sigma\left(u_{1}\right) v_{1} \nabla v_{1}-\sigma\left(u_{2}\right) v_{2} \nabla v_{2}\right) \nabla \bar{u} d x d \tau \\
& +\int_{0}^{t} \int_{\Omega}\left(\sigma\left(u_{1}\right) \beta\left(u_{1}\right) \nabla v_{1}-\sigma\left(u_{2}\right) \beta\left(u_{2}\right) \nabla v_{2}\right) \nabla \bar{u} d x d \tau \\
& \equiv I_{1}+I_{2} .
\end{aligned}
$$

Recall from our assumptions that $\nabla v_{1}, \nabla v_{2} \in\left[L^{\infty}\left(Q_{T}\right)\right]^{N} . I_{1}$ and $I_{2}$ can be estimated as follows:

$$
\begin{aligned}
\left|I_{1}\right| \leq \mid & \left|\int_{0}^{t} \int_{\Omega}\left(\sigma\left(u_{1}\right)-\sigma\left(u_{2}\right)\right) v_{1} \nabla v_{1} \nabla \bar{u} d x d \tau\right|+\left|\int_{0}^{t} \int_{\Omega} \sigma\left(u_{2}\right)\left(v_{1}-v_{2}\right) \nabla v_{1} \nabla \bar{u} d x d \tau\right| \\
& +\left|\int_{0}^{t} \int_{\Omega} \sigma\left(u_{2}\right) v_{2}\left(\nabla v_{1}-\nabla v_{2}\right) \nabla \bar{u} d x d \tau\right| \\
\leq & \left\{\left(\int_{0}^{t} \int_{\Omega}\left(\sigma\left(u_{1}\right)-\sigma\left(u_{2}\right)\right)^{2} d x d \tau\right)^{1 / 2}+\left(\int_{0}^{t} \int_{\Omega}\left(v_{1}-v_{2}\right)^{2} d c d \tau\right)^{1 / 2}\right. \\
& \left.+\left(\int_{0}^{t} \int_{\Omega}\left|\nabla v_{1}-\nabla v_{2}\right|^{2} d x d t\right)^{1 / 2}\right\}\left(\int_{0}^{t} \int_{\Omega}|\nabla \bar{u}|^{2} d x d \tau\right)^{1 / 2} \\
\leq & \left(c _ { 1 } \left(\int_{0}^{t} \int_{\Omega}^{2} d x d \tau+\int_{0}^{t} \int_{\Omega} \bar{v}^{2} d x d \tau\right.\right. \\
& \left.+\int_{0}^{t} \int_{\Omega}|\nabla \bar{v}|^{2} d x d t\right)+\frac{1}{4} \int_{0}^{t} \int_{\Omega}|\nabla \bar{u}|^{2} d x d \tau .
\end{aligned}
$$

Here we used the fact that $\sigma$ is Lipschitz continuous. Similarly,

$$
\left|I_{2}\right| \leq \frac{1}{4} \int_{0}^{t} \int_{\Omega}|\nabla \bar{u}|^{2} d x+c\left(\int_{0}^{t} \int_{\Omega} \bar{u}^{2} d x d \tau\right.
$$




$$
\left.+\int_{0}^{t} \int_{\Omega}|\nabla \bar{v}|^{2} d x d \tau\right)
$$

Clearly,

$$
\int_{0}^{t} \int_{\Omega} \sigma\left(u_{i}\right) \nabla v_{i} \nabla \xi d x=0 \text { for all } \xi \in L^{2}\left(0, t ; W_{0}^{1,2}(\Omega)\right)
$$

for all $0<t \leq T$ and for $i=1,2$.

Thus we obtain

$$
\int_{0}^{t} \int_{\Omega} \sigma\left(u_{2}\right)|\nabla \bar{v}|^{2} d x d \tau=-\int_{0}^{t} \int_{\Omega}\left(\sigma\left(u_{1}\right)-\sigma\left(u_{2}\right)\right) \nabla v_{1} \nabla \bar{v} d x d \tau .
$$

Consequently,

$$
\int_{0}^{t} \int_{\Omega}|\nabla \bar{v}|^{2} d x d \tau \leq c \int_{0}^{t} \int_{\Omega} \bar{u}^{2} d x d \tau
$$

By Poincaré's inequality,

$$
\begin{gathered}
\int_{0}^{t} \int_{\Omega} \bar{v}^{2} d x d \tau \leq c \int_{0}^{t} \int_{\Omega}|\nabla \bar{v}|^{2} d x d \tau \\
\leq c_{1} \int_{0}^{t} \int_{\Omega} \bar{u}^{2} d x d \tau .
\end{gathered}
$$

This immediately implies

By (3.3),

$$
\begin{aligned}
& \bar{u} \equiv \mathbf{0} . \\
& \bar{v} \equiv \mathbf{0} .
\end{aligned}
$$

Thus $u_{1} \equiv u_{2}, v_{1} \equiv v_{2}$. This completes the proof.

The above theorem is not very satisfactory because it requires that $\nabla v$ be bounded, which cannot be guaranteed by the existence theorem. Thus it is interesting to investigate when $\nabla v$ becomes bounded. We summarize our results in the following theorem.

THEOREM 3.2. Let the hypothesis of Theorem 2.3 be satisfied. 'Assume

(i) $U_{0} \in C^{0, \lambda}(\bar{\Omega})$ for some $0<\lambda<1$;

(ii) $\frac{\partial}{\partial x_{i}} B \in L^{\infty}\left(0, T ; C^{0, \lambda}(\bar{\Omega})\right)$ for each $i$;

(iii) $N=2$;

(iv) $\sigma$ is Lipschitz continuous.

Then there is a $\lambda_{1} \in(0,1)$ such that $\frac{\partial}{\partial x_{i}} v \in L^{\infty}\left(0, T ; C^{0, \lambda_{1}}(\bar{\Omega})\right)$ for $i=1,2$.

PROOF. Set

$$
\psi=v-B
$$

Then for a.e. $t \in[0, T]$, we have

$$
\int_{\Omega} \sigma(u(x, t)) \nabla \psi(x, t) \nabla \xi(x) d x=-\int_{\Omega} \sigma(u(x, t)) \nabla B(x, t) \nabla \xi(x) d x
$$

for all $\xi \in W_{0}^{1,2}(\Omega)$. That is to say, we view (2.34) as a family of elliptic equations. Then for a.e. $t$ in $(0, T)$ we appeal to a result due to Meyers $[8, \mathrm{p} .36]$ to conclude that there is a positive number $c$ depending only on $m, M$ in (H5) and on $\Omega$ such that 


$$
\|\nabla \psi(x, t)\|_{p, \Omega} \leq c\|\nabla B(x, t)\|_{p, \Omega} \leq c_{1}
$$

for some $p>2$. Thus $v \in L^{\infty}\left(0, T ; W^{1, p}(\Omega)\right)$. Since $u$ satisfies $(2.54)$ and $p>N=2$, we may invoke the classical regularity theory for linear parabolic equations [5, pp. 181-204] to get

$$
u \in H^{\lambda, \lambda / 2}\left(\overline{Q_{T}}\right) \text { for some } \lambda>0 .
$$

It immediately follows that $u \in L^{\infty}\left(0, T ; C^{0, \lambda}(\bar{\Omega})\right)$. Recall that $\sigma$ is Lipschitz continuous. Hence $\sigma(u(x, t)) \in C^{0, \lambda}(\bar{\Omega})$ for all $t \in[0, T]$. We are in a position to apply a result in [6, p. 210] to (3.4), thereby establishing

$$
\begin{aligned}
& |\psi(\cdot, t)|_{1, \lambda} \leq c_{1}+c_{2} \mid \sigma\left(\left.u(\cdot, t) \nabla B(\cdot, t)\right|_{0, \lambda}\right. \\
& \leq c \text { for some } 1>\lambda>0 \text { and for all } t \in[0, T]
\end{aligned}
$$

The proof is complete.

Combining Theorems 3.1 and 3.2 yields the following:

THEOREM 3.3. Let the assumptions of Theorem 3.2 hold. Assume that $\Theta(s)=s$. Then there exists a unique solution to (1.1).

ACKNOWLEDGEMENT. This work was supported in part by the National Science Foundation under grant No. DMS-9101382.

\section{REFERENCES}

1. CIMATTI, G., Existence and uniqueness for the equations of the Joule-Thomson effect, Applicable Anal. 41 (1991), 131-144.

2. CIMATTI, G., Existence of weak solutions for the nonstationary problem of the Joule heating of a conductor, Ann. Mat. Pura Appl., (To appear).

3. SHI, P.; SHILLOR, M. \& XU, X., Existence of a solution to the Stefan problem with Joule's heating, J. Differential Equations (To appear).

4. EVANS, L.C., Weak Convergence Methods for Nonlinear Partial Differential Equations, AMS, Providence, Rhode Island, 1990.

5. LADYZENSKAJA, O.A.; SOLONNIKOV, V.A. \& URALCEVA, N.N., Linear and quasilinear equations of parabolic type, Tran. of Math. Monographs 23, AMS, Providence, Rhode Island, 1968.

6. GILBARG, D. \& TRUDINGER, N.S., Elliptic Partial Differential Equations of Second Order, 2nd Ed., Springer-Verlag, Berlin, 1983.

7. ODEN, J.T., Qualitative Methods in Nonlinear Mechanics, Prentice Hall, Inc. Englewood Cliffs, New Jersey, 1986.

8. BENSOUSSAN, A.; LIONS, J.L. \& PAPANICOLAOU, G., Asymptotic Analysis for Periodic Structures, North-Holland, Amsterdam, 1978. 


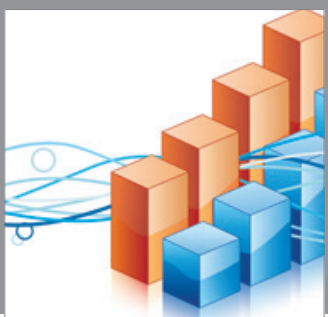

Advances in

Operations Research

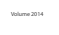

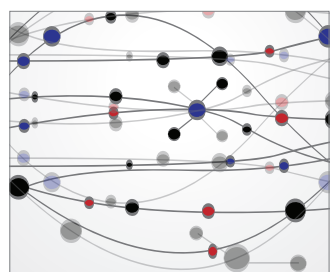

\section{The Scientific} World Journal
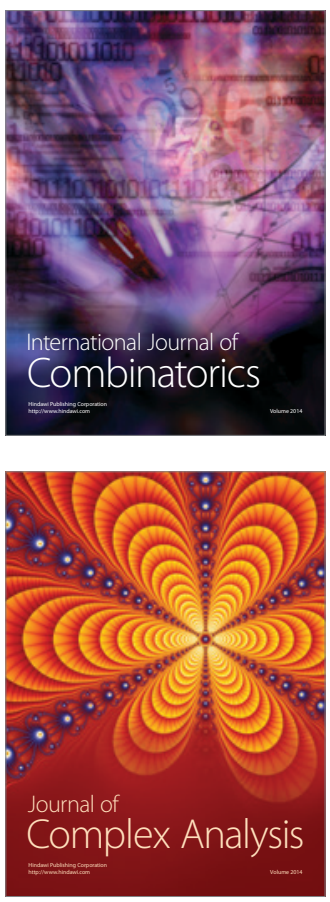

International Journal of

Mathematics and

Mathematical

Sciences
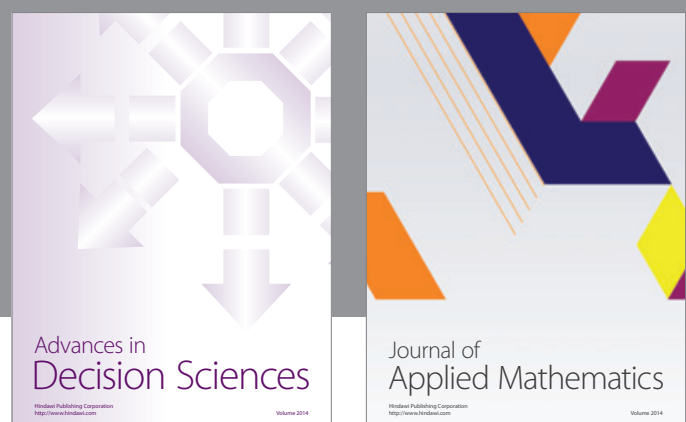

Journal of

Applied Mathematics
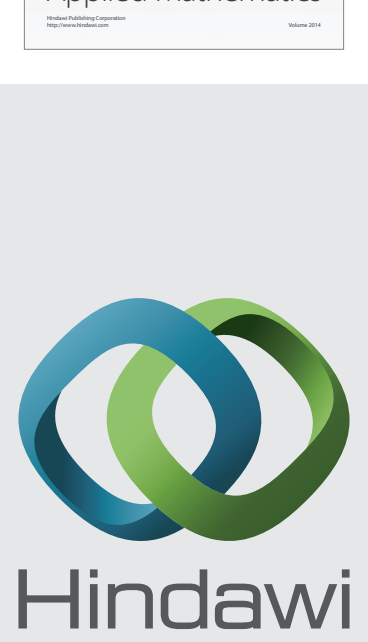

Submit your manuscripts at http://www.hindawi.com
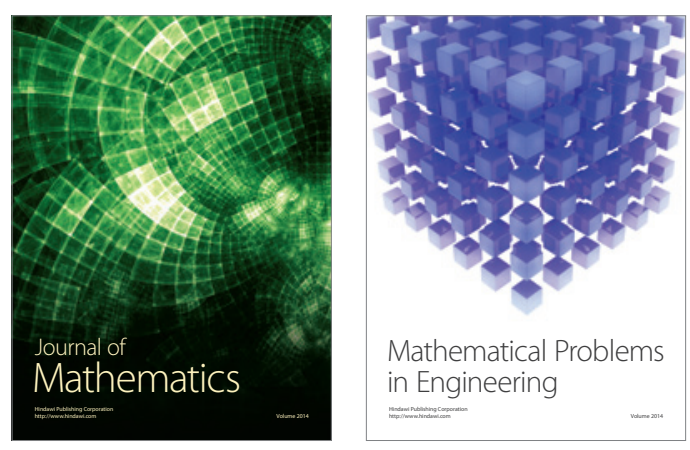

Mathematical Problems in Engineering
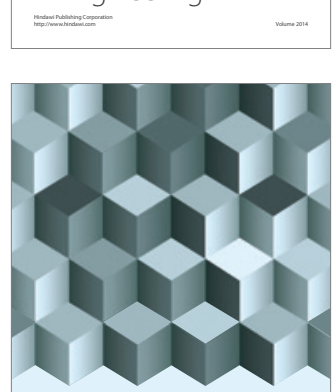

Journal of

Function Spaces
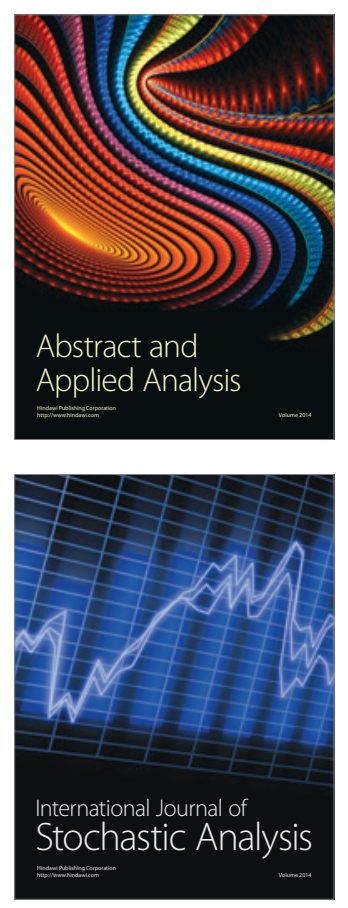

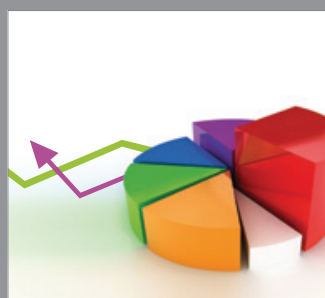

ournal of

Probability and Statistics

Promensencen
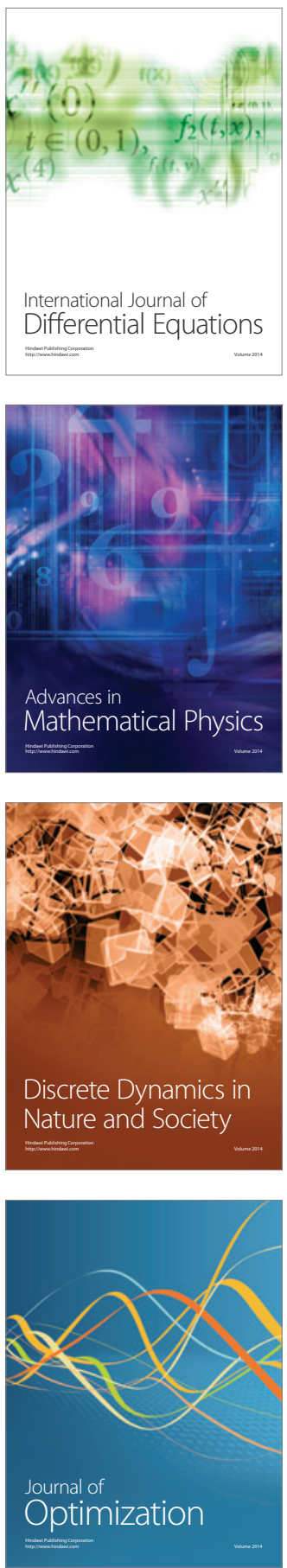